\title{
Ausencia de habilidades gerenciales para una cultura de innovación universitaria en instituciones de Colombia y Venezuela
}

\section{Zoraima Aurelia Donawa Torres* Willington German Gámez Araujo**}

Fecha de recibido: 25 de julio de 2017 Fecha de aprobado: 2 de mayo de 2018

Para citar: Donawa Torres, Z. A, \& Gámez Araujo, W. G. (2019). Ausencia de habilidades gerenciales para una cultura de innovación universitaria en instituciones de Colombia y Venezuela. Universidad \& Empresa, 21(36), 8-35. DOI: http://dx.doi.org/10.12804/revistas.urosario.edu.co/empresa/a.5961

Licenciada en Ciencias Políticas y Administrativas. Magíster en Gerencia de Empresas. Doctora en Ciencias Gerenciales y Postdoctora en Gerencia de las Organizaciones. Profesora e investigadora, categoría agregado, Instituto Universitario de Tecnología de Cabimas, Zulia, Venezuela. Correo electrónico: zdonawa62@hotmail.com. Calle Vargas N43, casa Mis Arcángeles entre calles Colombia y San Benito Cabimas Edo. Zulia. Venezuela.

** Coordinador de la Facultad de Ingeniería Industrial, Universidad Antonio Nariño sede Santa Marta, Colombia. Correo electrónico: willingthongamez@gmail.com 


\section{Resumen}

El objetivo fundamental de esta investigación fue identificar las habilidades gerenciales para una cultura de innovación universitaria en instituciones de Colombia y Venezuela. Para cumplir con el objetivo, se estudiaron las bases teóricas de los autores Horth y Buchner (2009), Dyer, Gregersen y Christensen (2012) y Thomas (2010), entre otros. La metodología utilizada fue bajo el paradigma de investigación positivista y la investigación fue descriptiva con un diseño no experimental, transeccional y de campo. La población estuvo constituida por 60 docentes de cada universidad. Como instrumento para la recolección de datos se elaboró un cuestionario utilizando las categorías de respuestas de tipo Likert de 33 ítems, validado por siete expertos en el área. La confiabilidad del instrumento fue determinada por el método Alfa de Cronbach y presentó un 0,94 de confiabilidad. Los resultados de la investigación permiten evidenciar un bajo porcentaje tanto en las habilidades necesarias para ser innovadores, como en las habilidades de descubrimiento, por lo que se concluye la ausencia de las habilidades gerenciales para una cultura de innovación universitaria en instituciones de educación superior de Colombia y Venezuela.

Palabras clave: habilidades, habilidades gerenciales, cultura de innovación.

\section{Absence of Managerial Skills for a Culture of University Innovation in Institutions of Colombia and Venezuela Abstract}

The main objective of this research was to identify management skills for a culture of university innovation in higher education institutions in Colombia and Venezuela. To meet the objective, we studied the theoretical bases of the authors Horth and Buchner (2009), Dyer, Gregersen and Christensen (2012) and Tomás (2010), among others. The methodology used was under the positivist research paradigm, the research was descriptive with a non-experimental, transsectional and field design. The population was constituted by 60 teachers of each university. As a tool for data collection, a questionnaire was developed using the 33-item Likert response categories, validated by seven experts in the area. The reliability of the instrument was determined by the Cronbach's Alpha method with a reliability of 0.94 . The research results show a low percentage of both the skills needed to be innovative and the discovery skills, concluding that there is a lack of managerial skills for a culture of university innovation in higher education institutions in Colombia and Venezuela.

Keywords: Abilities, management skills, culture of innovation.

\section{Ausência de habilidades gerenciais para uma cultura de inovação universitária em instituições da Colômbia e a Venezuela Resumo}

O objetivo fundamental desta pesquisa foi identificar as habilidades gerenciais para uma cultura de inovação universitária em instituições da Colômbia e a Venezuela. Para cumprir com o objetivo, estudaram-se as bases teóricas dos autores Horth e Buchner (2009), Dyer, Gregersen e Christensen (2012) e Tomás (2010), entre outros. A metodologia utilizada foi sob o paradigma de pesquisa positivista, a pesquisa foi descritiva com um desenho não experimental, transversal e de campo. A amostra esteve constituída por 60 professores de cada universidade. Como instrumento para a colheita de dados elaborou-se um questionário empregando as categorias de respostas de tipo Likert de 33 itens, validado por sete expertos na área. A confiabilidade do instrumento foi determinada pelo método Alfa de Cronbach apresentando um 0.94 de confiabilidade. Os resultados da pesquisa permitem evidenciar uma baixa percentagem tanto nas habilidades necessárias para serem inovadores, quanto nas habilidades de descobrimento, pelo que se conclui a ausência das habilidades gerenciais para uma cultura de inovação universitária em instituições de educação superior da Colômbia e da Venezuela.

Palavras-chave: habilidades, Habilidades gerenciais, Cultura de inovação. 


\section{Introducción}

Las organizaciones deben hacerle frente al fenómeno de la globalización, pues este obliga a las empresas a crear una cultura de innovación en la que se desarrollen las habilidades gerenciales necesarias para responder a sus retos, así como un pensamiento innovador que debe contribuir en el proceso de las actividades propias de la enseñanza y el aprendizaje.

Una cultura de la innovación en las organizaciones de educación superior alienta a las personas a trabajar en innovaciones, porque todos están acostumbrados a sugerir mejoras, modificaciones continuas y replanteamientos institucionales; así, se crea un ambiente al interior de las organizaciones en el que todas las personas se sienten parte activa de los procesos. En este sentido, la investigación se trazó como objetivo general determinar las habilidades gerenciales para una cultura de innovación universitaria en instituciones de Colombia y Venezuela, y como objetivos específicos, identificar las habilidades gerenciales necesarias para ser innovadores y distinguir la presencia de habilidades gerenciales de descubrimiento.

La investigación se estructura de la siguente manera. En primer lugar, expone un marco teórico que describe el comportamiento de las variables y después, describe el marco metodológico utilizado, los resultados y las conclusiones sobre el estudio.

\section{Revisión de literatura}

\subsection{Habilidades gerenciales}

En tanto que los autores Danilov y Skatkin (1985, citados por Madrigal, 2009) expresan que la habilidad es la capacidad adquirida por el hombre de utilizar de manera creativa sus conocimientos durante el proceso de actividades educativas, Chiavenato (2009) afirma que el significado de habilidad es utilizar y aplicar el conocimiento, ya sea para resolver problemas o situaciones, crear o innovar; además, explica habilidad es la transformación del conocimiento en resultado y que las personas requieren de muchas habilidades para trabajar en una organización. 
Por su parte, Madrigal (2009) considera que las habilidades de un gerente se miden en función de su capacidad y aptitud para administrar, tomar medidas, negociar y arreglar problemas. Distingue el autor que la enseñanza y el aprendizaje de las habilidades gerenciales se cultivan y educan, se aprenden y se desarrollan mediante cursos, talleres, especialidades, maestrías y doctorados en el área. Así mismo, señala que entre las habilidades que un gerente debe dominar, en primer lugar está la comunicación, saber tomar decisiones y estar consciente del riesgo que cada una conlleva, tener creatividad para innovar, improvisar y planear, ser líder, saber manejar el tiempo y, sobre todo, ser asertivo.

Los autores Belker y Topchik (2007) advierten que el gerente debe ser el encargado de lograr que los colaboradores se autodirijan, que realicen sus funciones con gusto y no solo porque lo tengan que hacer, por lo tanto, deben enfocarse en su relación con los colaboradores y luego con la tarea que deben desempeñar; además, necesitan confiar en ellos.

Hirt y Ferrel (2010) afirman que todos los gerentes deben tener habilidades conceptuales definiéndolas como las que incluyen la capacidad para pensar en forma creativa; ellos sustentan esta afirmación acotando que las investigaciones científicas recientes han arrojado que es posible aprender a pensar de modo creativo, lo cual es la base del desarrollo de muchos productos e ideas innovadoras. Por su parte, Whetten y Cameron (2005) consideran que el desarrollo de habilidades gerenciales es determinante para el éxito de la organización y advierten que las destrezas directivas son como un vehículo a través del cual la estrategia y la práctica de herramientas administrativas llegan a conseguir el resultado esperado.

En el mismo orden de ideas, Whetten y Cameron (2011) acotan que las habilidades gerenciales son el vehículo mediante el cual la estrategia y la práctica de la administración, las herramientas y las técnicas, los atributos de la personalidad y el estilo trabajan para producir resultados eficaces dentro de administración de las organizaciones. Cabe destacar que la innovación es la herramienta específica de los gerentes innovadores, ya que es la habilidad que permite al gerente explotar el cambio como una oportunidad para un negocio diferente, es la acción de dotar a los recursos con una nueva capacidad de producir riqueza, es la creación de un recurso, afirma. (Ducker, 2002). 
De igual forma, Chiavenato (2009) afirma que es importante considerar que en la gestión gerencial, la administración de la innovación, tanto de procesos como de productos o servicios, incluye un apoyo intensivo a la invención (el acto de descubrir) y a la aplicación (el acto de usar). También señala que la innovación se refiere al desarrollo de nuevas ideas y afirma que se requieren las habilidades gerenciales para la creación de nuevos ambientes de trabajo que estimulen la creatividad y el surgimiento de nuevas ideas a través de las personas.

No obstante, los autores Koontz, Weihrich y Cannice (2012) distinguen que la creatividad se refiere a la habilidad y el poder de desarrollar nuevas ideas; la innovación, en cambio, significa el uso de estas ideas. Explican que en una organización esto puede significar un nuevo producto, un nuevo servicio o una nueva forma de hacer las cosas. Aunque este análisis se concentra en el proceso creativo, supone que las organizaciones no sólo generan nuevas ideas, sino que las traducen en aplicaciones prácticas. Por su parte, Robbins y Judge (2013) afirman que la innovación es la clase más especializada de cambio, ya que las organizaciones innovadoras utilizan dicha disciplina como una herramienta para reinventar su futuro, mediante la generación de ideas y la apertura de nuevos mercados, originada por la introducción de nuevos productos y servicios.

\subsection{Habilidades de pensamiento innovador}

En palabras de Ohmae (2007), el pensamiento estratégico es una combinación de los métodos analíticos y la elasticidad mental que los gerentes deben utilizar con el propósito de lograr obtener ventajas competitivas. Del mismo modo, Robert (2006) considera que el desarrollo del pensamiento estratégico en el gerente de organización lleva a la creación de un equipo directivo con una visión compartida de futuro, un compromiso personal, una identificación con el proyecto y con sus estrategias, y un sentimiento profundo de autoridad y paternidad por parte de todas las personas claves de la organización.

En este sentido, Friedmann (citado por Robert, 2006) afirma que la innovación surge del pensamiento estratégico reflejado en el gerente que pretende conseguir lo que desea de la mejor forma, venciendo todas las dificultades, aplicando su juicio de valor basado en su experiencia con el propósito de establecer las direcciones futuras, desarrollando su creatividad y abriendo paso hacia la innovación. 
Mintzberg (1998) señala que el pensamiento estratégico es la utilización de la intuición y la creatividad para crear una perspectiva integrada de la empresa, en tanto que Morrissey (1996) explica que el pensamiento estratégico individual incluye la aplicación del juicio basado en la experiencia para determinar las direcciones futuras, mientras que el pensamiento estratégico de las empresas es la coordinación de mentes creativas dentro de una perspectiva que le permita al negocio avanzar hacia el futuro de una manera satisfactoria para todos. A nivel gerencial, Morrisey (1996) afirma que el pensamiento estratégico a nivel individual comprende el entendimiento holístico de la organización y su ambiente, la imaginación, la creatividad y la visión de futuro; además, sostiene que lo primero que debe buscarse es el claro entendimiento del carácter particular de cada elemento de una coyuntura dada y después hacer el más completo uso posible del poder del cerebro para reestructurar esos elementos en la forma más ventajosa.

En este sentido, Barba (2011) expresa que Ansoff (1967) consideró que cuando un gerente entiende el ambiente y reconoce su condición de cambio constante, puede tomar las decisiones correctas liderando las organizaciones hacia el futuro.

Saiz (2002, citado por Lara, 2012) señala que el pensamiento creativo es generador de ideas y alternativas, de soluciones nuevas y originales; permite comprender, inventar y establecer nuevas y personales conexiones entre lo que se sabe y lo que se aprende, dando paso a una configuración del conocimiento de carácter significativo, que está íntimamente ligado al pensamiento crítico, el cual puede verse favorecido a través de la adquisición de estrategias de procesamiento analógico orientadas a la generación de nuevas ideas.

Estas consideraciones permiten inferir que la gerencia universitaria debe considerar, en el pensamiento creativo y estratégico, las habilidades de pensamiento innovador. Resulta oportuno enunciar que los autores Horth y Buchner (2009) establecen seis habilidades necesarias para el pensamiento innovador apreciadas en la figura 1: 


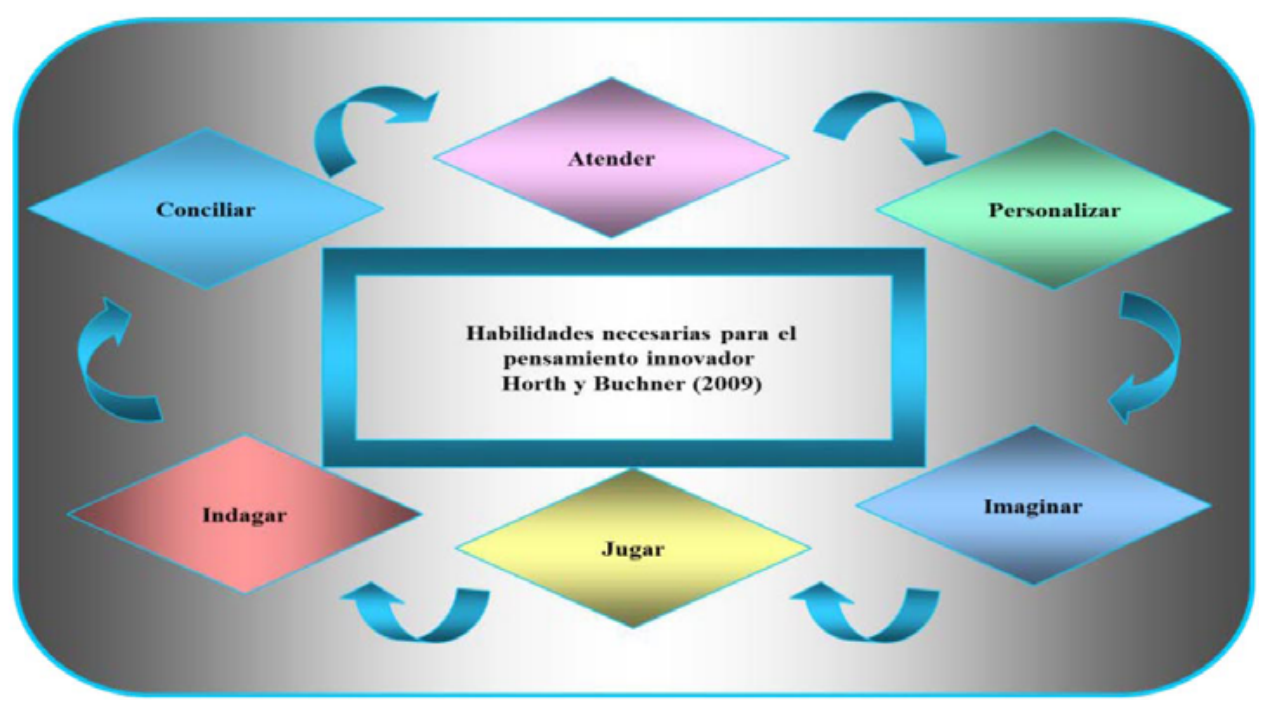

Figura 1. Habilidades necesarias para el pensamiento innovador Fuente: elaborada por los autores.

\subsubsection{Prestar atención}

Explican los autores que la habilidad de prestar atención, es decir, la capacidad de darse cuenta de algo que ha pasado desapercibido, consiste en examinar una situación en mayor profundidad, lo que implica ser un observador agudo, que percibe los detalles e identifica nuevos patrones. Para prestar atención es necesario frenar la marcha temporalmente a fin de ser reflexivo a la hora de comprender la situación. Es necesario evaluar distintos puntos de vista y múltiples datos, así como observar y escuchar desde una nueva perspectiva.

Hargreaves y Fink (2008) consideran que los líderes en organizaciones de educación superior con cultura de innovación, preservan, protegen y fomentan el aprendizaje profundo y amplio para todos, en unas relaciones de atención a los demás. En este sentido, las primeras impresiones y suposiciones no conforman la imagen total y por ello no conducen a una evaluación ajustada o a la mejor solución.

\subsubsection{Personalizar}

Horth y Buchner (2009) distinguen que en el ámbito del pensamiento innovador, personalizar es un proceso doble, pues consiste en aprovechar los amplios conocimientos y experiencias personales y comprender al consumidor de forma personal y profunda. Así mismo, consideran que la capacidad de aprovechar experiencias y emociones persona- 
les sin relación aparente introduce perspectivas nuevas en los retos. Puntualizan que la personalización aprovecha los intereses, pasatiempos o aficiones y los aplica al trabajo, buscando siempre la manera de que ideas, patrones o experiencias de las personas puedan contribuir a enriquecer su labor. El conocimiento profundo de los mismos conduce a ideas, patrones y experiencias nuevas que alimentan la innovación. En palabras de Hargreaves y Fink (2008), mediante la personalización se otorga más importancia a esta experiencia con el fin de extraer conclusiones de la misma.

\subsubsection{Imaginar}

Esta habilidad según los autores, es la capacidad de procesar la información. Explican que las palabras por sí solas no bastan para extraer el sentido de informaciones complejas o muy vastas. Imaginar es un buen modo de abordar esta información y darle sentido. Según Horth y Buchner (2009), las imágenes, historias, impresiones y metáforas son herramientas poderosas para describir situaciones, construir ideas y comunicar de manera eficaz; estas ideas pueden provenir del interior o del exterior de la organización. Por otra parte, la creatividad es, según Daft (2015), la generación de ideas novedosas que pueden satisfacer necesidades percibidas o responder a las oportunidades.

Lara (2012) define la creatividad como la elaboración mental, autónoma y transformativa del sujeto y los objetos, en la que intervienen procesos cognitivo-afectivos para la generación y el desarrollo de ideas nuevas, relevantes y pertinentes, para la formulación y solución de retos que responden a sus intereses y expectativas. Del mismo modo, Waisburd (2009) identifica la imaginación como una herramienta poderosa para activar el pensamiento creativo, ya que, como afirmaría Einstein, "es más importante la imaginación que el conocimiento, ya que la imaginación no tiene límites y el conocimiento si los tiene”.

\subsubsection{Jugar de manera reflexiva}

Según Horth y Buchner (2009), el pensamiento empresarial y el trabajo rutinario pueden convertirse en un proceso rígido, por ello la innovación requiere romper algunas reglas, crecer y divertirse. Del mismo modo, cuando se genera conocimiento y experiencia mediante formas no tradicionales, como la libre exploración, la improvisación, la experimentación, la construcción de prototipos de manera rápida y ligera, o la exploración de los límites, el trabajo parece un juego pero los resultados que se obtienen son serios. 
Las organizaciones deben despertar la creatividad en las personas para reforzar su cultura de innovación; esta es un rasgo de la personalidad que comprende la capacidad para desprenderse de pensamientos ligados a hábitos y generar ideas novedosas y útiles. La creatividad produce innovación y ésta es el alma de cada vez más corporaciones, tal como lo afirman autores como Ivancevich, Konopaske y Matteson (2006).

\subsubsection{Indagar en colaboración con otras personas}

A juicio de los autores señalados, las innovaciones muy pocas veces son obra de un "genio solitario", sino que se obtienen a través de un intercambio de ideas reflexivo e imparcial. La indagación en colaboración es un proceso de diálogo sostenido y eficaz con las partes interesadas. Aprovechar la oportunidad que supone contar con múltiples partes interesadas y diferentes puntos de vista puede generar una mayor complejidad, pero también es fuente de grandes oportunidades. Se deben plantear preguntas que inviten a la indagación, recurriendo al pensamiento crítico sin esperar respuestas inmediatas.

Es importante acotar que dentro de las habilidades gerenciales para una cultura de innovación se destacan los comportamientos relacionados con el proceso de transmitir información, es decir, para lograr la innovación se debe escuchar las ideas, sugerencias y opiniones de los empleados, consultando decisiones, ofreciendo y solicitando retroalimentación (Rodríguez, 2008).

Cabe destacar que la indagación permite a la gerencia de las organizaciones actualizarse sobre las nuevas tecnologías e innovaciones. En este sentido, Murcia (2010) explica que la creatividad es una habilidad y una actitud que tiene permanente vigencia; además, sugiere que, ante la gran cantidad de cambios acelerados que se dan en la actualidad, es vital mantener una actitud creativa continua, reconociendo que muchas de las cosas que funcionaron en el pasado no son válidas en el presente.

\subsubsection{Conciliación}

Horth y Buchner (2009) afirman que la innovación requiere desechar el pensamiento exclusivo y contemplar el todo como una oposición inclusiva y abierta a una tercera (o cuarta, o quinta) solución. Expresan que conciliar permite vivir con la paradoja y la contradicción, y resolverlas; además, sostienen que, a diferencia del análisis tradicional del pensamiento empresarial, que requiere la división de los problemas en partes separa- 
das, hechos conocidos y suposiciones actuales, conciliar tiene que ver con la síntesis, la integración y la posibilidad.

Mediante lo que se conoce como razonamiento abductivo, se pueden establecer conexiones intuitivas entre informaciones aparentemente no relacionadas y comenzar a extraer orden del caos. Por su parte, Cárdenas (2011) expresa que el razonamiento abductivo es un tipo de inferencia cuyos vínculos con la actividad mental no son exclusivos, pues tiene nexos estrechos con la acción humana.

Bajo este contexto teórico, centrada en los autores Horth y Buchner (2009) y complementada con otros autores, esta investigación se encamina a alcanzar el objetivo de identificar las habilidades gerenciales necesarias para ser innovadores en instituciones de educación superior de Colombia y Venezuela.

\subsection{Habilidades gerenciales de descubrimiento}

Cuando los gerentes innovadores descubren ideas pioneras, se comportan de manera similar, pues cuentan con cinco habilidades denominadas por Dyer, Gregersen y Chrsitensen (2012) como el ADN del innovador. Ellos distinguen los gerentes innovadores porque piensan diferente, es decir, sus mentes se destacan por asociar ideas que, a priori, no guardan relación para producir ideas originales. Así mismo, actúan diferente, pues todos son grandes interrogadores, que plantean preguntas que ponen en tela de juicio el statu quo imperante.

Agregan los autores que algunos gerentes innovadores observan el mundo con una agudeza fuera de lo común, otros trabajan en colaboración con las personas más diversas que puedan encontrar y otros colocan la experimentación en el centro de su actividad innovadora, mezclando las acciones de preguntar, observar, colaborar y experimentar y dando origen a un pensamiento asociativo que genera nuevos negocios, productos, servicios o procesos. En este sentido, infieren que la creatividad no es un atributo genético ni una mera habilidad cognitiva, sino que las ideas creativas parten de habilidades conductuales que también se pueden adquirir para catalizar ideas innovadoras en cada persona.

Es oportuno mencionar que López (2016) define la creatividad como una capacidad potencialmente presente de las más variadas maneras, en cada persona y en todos los 
grupos humanos y culturas; además, afirma que está ligada a todos los aspectos de la experiencia y es una poderosa herramienta para plantear y resolver problemas, diseñar el presente y pensar en el futuro.

Por su parte, Whetten y Cameron (2011) señalan que la creatividad mediante la imaginación se refiere a la creación de nuevas ideas, grandes avances y métodos radicales para la solución de problemas. Los individuos que buscan la creatividad de esta forma suelen ser experimentadores, especuladores y emprendedores, y definen la creatividad como exploración, innovación de productos o desarrollo de posibilidades.

Dyer, Gregersen y Christensen (2012) afirman que los innovadores tienen la capacidad de utilizar ambos hemisferios del cerebro, lo cual les permite disfrutar de cinco habilidades de descubrimiento: asociar, cuestionar, observar, experimentar y relacionarse (figura 2).

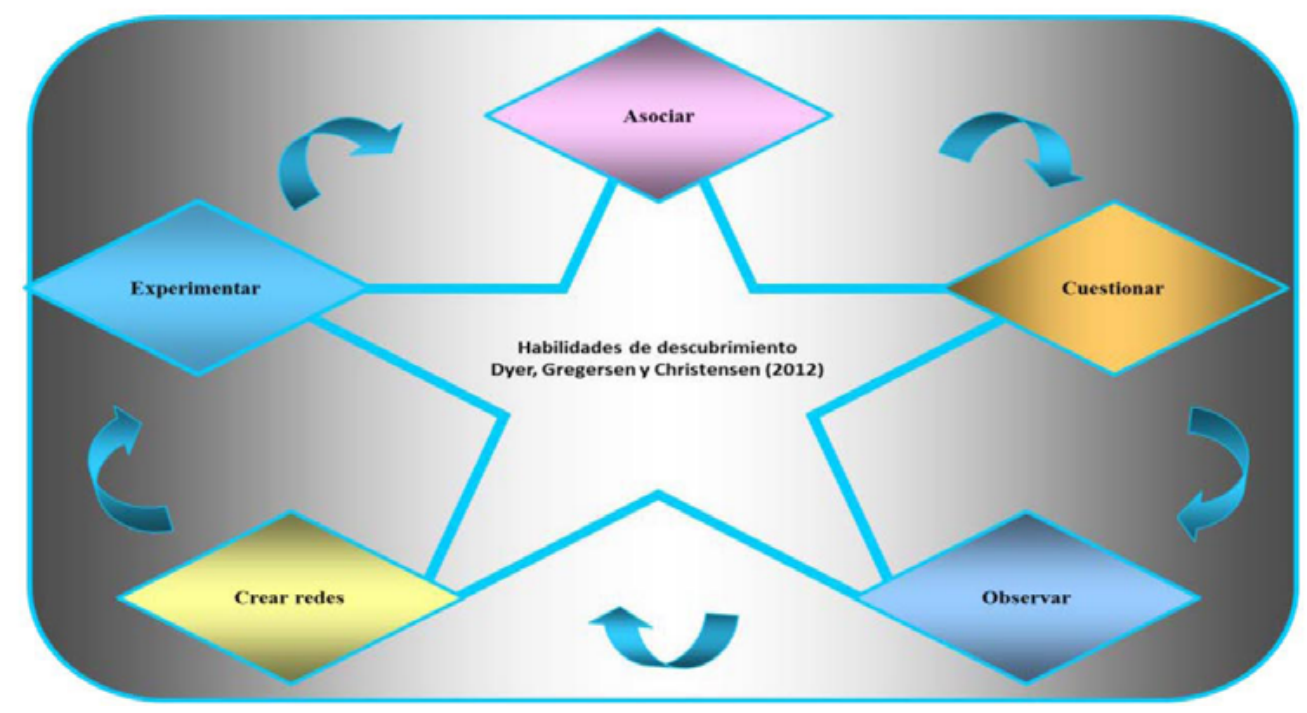

Figura 2. Habilidades de descubrimiento

Fuente: elaborada por los autores.

\subsubsection{Habilidad de descubrimiento: asociación}

Esta habilidad, que a criterio de los autores está en el núcleo del ADN del innovador, consiste en hacer conexiones inesperadas entre distintas áreas de conocimiento, diversos sectores industriales e incluso geografías. Es una habilidad que suele darse por sentada entre los innovadores. Según los autores, las personas creativas poseen la capacidad de conectar sus experiencias pasadas y de sintetizarlas creando otras nuevas. 
Por su parte, Gómez (2013) afirma que, aun cuando no hay completo acuerdo acerca de las características precisas para definir el pensamiento creativo, los equipos de personas con diferentes tipos de pensamiento e inteligencias suelen tener resultados creativos.

Pérez (2013) explica que en el contexto educativo, las universidades y el docente, como entes que persiguen la transformación del individuo, deben promover un pensamiento creativo en los estudiante que dé como fruto soluciones a necesidades de la persona y la sociedad. En este sentido, afirma que la creatividad debe estar plasmada de manera normativa y activa transversalmente desde el currículum hasta la evaluación. Estar presente en cada uno de sus actores. Ser materia prima, técnica, proceso y producto.

Por su parte, Dyer, Gregersen y Christensen (2012) explican algunos factores que influyen en la dinámica de las asociaciones y que describen la dinámica del pensamiento de los innovadores disruptivos, estas son:

- La creación de combinaciones extrañas: es la acción que realizan los innovadores cuando intentan reunir ideas en apariencia inconexas para formar combinaciones sorprendentemente exitosas. En este sentido, se preguntan continuamente: "¿qué pasaría si se combina esto con eso, o esto con esto y eso con aquello?". Piensan diferente uniendo sin temor combinaciones de ideas poco comunes.

- Acercarse y alejarse de la imagen: en esta acción, los gerentes innovadores muestran la capacidad de hacer dos cosas a la vez: se sumergen profundamente en los detalles para entender los sutiles matices de la experiencia particular del cliente y vuelan alto para ver cómo encajan los detalles en la visión global. La síntesis de estas dos perspectivas a menudo desencadena sorprendentes asociaciones.

- Pensamiento Lego: los autores explican que si los innovadores tienen algo en común es que a todos les encanta coleccionar ideas, como los niños coleccionan Legos. En este sentido, consideran que recopilar multitud de ideas de gran número de fuentes diferentes genera el mejor de los mundos de la innovación. Esto significa que, cuanto más conocimiento, experiencia o ideas de las disciplinas más variadas se añadan a la reserva global de ideas, mayor será la diversidad de ideas que se puedan construir. 


\subsubsection{Habilidad de descubrimiento: cuestionamiento}

El cuestionamiento es el catalizador creativo para otros comportamientos de descubrimiento, como la observación, la creación de redes y la experimentación. Los innovadores con esta habilidad desarrollan su trabajo haciendo preguntas que invitan a la reflexión con el objetivo de entender mejor qué es una cosa y lo que podría ser. Ignoran las preguntas prudentes y optan por aquellas disparatadas, que desafían el statu quo y que a menudo cuestionan el poder establecido con una intensidad y frecuencia poco habituales. Estas habilidades de cuestionamiento se desarrollan cuando una persona:

- Se involucra en tormentas de preguntas: distinguen los autores que la tormenta de ideas, un proceso en el que se reúne el gerente con un equipo para plantear soluciones a un problema. La tormenta de preguntas es similar, pero en lugar de centrarse en las soluciones, se proponen preguntas sobre el problema; estas les ayudan a profundizar en el problema y las más habituales que se deben hacer son las siguientes: ¿qué hay?, ¿cuál fue la causa?, ¿por qué?, ¿por qué no?, ¿qué pasaría si...?

- Cultiva el pensamiento indagatorio: los autores advierten que cuando se identifican problemas o retos, a menudo se describen con afirmaciones, sin embargo, se ha descubierto que la reconversión de las afirmaciones en preguntas no solo ayuda a pulir la definición del problema, sino que suscita una mayor responsabilidad personal con respecto a los problemas e incita a adoptar pasos más activos en la búsqueda de respuestas.

- Dispone de un cuaderno solo para preguntas: consideran los autores que para crear una sustanciosa reserva de preguntas, se han de tomar de vez en cuando un tiempo para formularlas y se debe dedicar unos minutos para reflexionar sobre las siguientes cuestiones: ¿en qué tipo de preguntas centrarse?, ¿qué preguntas cuestionan el statu quo?, ¿qué preguntas generan potentes respuestas emocionales?

\subsubsection{Habilidad de descubrimiento: observación}

A juicio de los autores, la mayoría de los innovadores son intensos observadores del mundo que los rodea y cuando observan el funcionamiento de las cosas, a menudo se dan cuenta de aquello que no funciona. Además, cuando se involucran en este tipo de observaciones, empiezan a establecer conexiones entre datos aparentemente no relacionados que pueden 
generar ideas empresariales inusuales. Para desarrollar esta habilidad de innovador, Dyer, Gregersen y Christensen (2012) emiten los siguientes consejos:

- Observar al cliente: el gerente puede perfeccionar y afilar las habilidades de observación organizando excursiones regulares para mirar con atención cómo ciertos consumidores utilizan los productos o servicios de la organización; también se puede observar a personas reales en situaciones de la vida real, buscando las cosas que les hacen la vida más fácil y aquellas que les complican la vida.

- Observar a las empresas: se elige una empresa a la que observar y seguir, podría tratarse de una compañía por la que se siente admiración o una empresa de reciente creación con un innovador modelo de negocio. A medida que se vaya aprendiendo cosas sobre ella, se pueden hacer las siguientes preguntas: ¿hay alguna idea que puede transferir y adaptar a esta organización?, ¿cómo puede esta estrategia, táctica o actividad afectar al trabajo, a la organización o a la vida de sus miembros?

- Observar cualquier cosa que llame nuestra atención: explican los autores que se debe dedicar al menos diez minutos al día para observar algo intensamente, tomar notas y luego intentar resolver cómo lo que se está viendo puede conducir a una nueva estrategia, producto, servicio o proceso de producción.

\subsubsection{Habilidad de descubrimiento: 'networking' o creación de redes}

Los autores consideran que, con frecuencia, pensar de forma creativa genera la conexión de ideas del área de conocimiento propias con las de quienes juegan en campos diferentes o están fuera de la esfera de influencia. Los innovadores adquieren una perspectiva radicalmente distinta cuando dedican tiempo y energía a encontrar y probar ideas a través de una red integrada por individuos diversos. De igual manera, el principio básico de la creación de redes de ideas es construir un puente entre diferentes áreas de conocimiento mediante la interacción con las personas. Del mismo modo, Dyer, Gregersen y Christensen (2012) contribuyen con unos consejos para que los gerentes innovadores desarrollen la habilidad para establecer redes de contactos: 
- Ampliar la diversidad de la red de contactos: se puede hacer una lista de las diez personas con las que se hablaría si se intenta obtener o pulir una nueva idea; es conveniente que sean de edades, nacionalidades y clases sociales diferentes.

- Establecer un plan de almuerzos con la red de contactos: se debe organizar como mínimo un almuerzo a la semana con personas de distintos entornos.

- Planificar la asistencia al menos a dos conferencias el próximo año: se sugiere seleccionar y ver una conferencia sobre un tema relacionado con el área de especialización y una segunda que no tenga nada que ver.

- Intercambiar formación con expertos: se recomienda buscar especialistas de profesiones, sectores, industrias y países diferentes, y acudir a sus reuniones y sesiones de formación para experimentar su trabajo y su mundo por espacio de un mes, con el fin de adquirir nuevas perspectivas sobre sus respectivos mundos, así como nuevas formas para cuestionar los supuestos fundamentales en el sector del otro.

\subsubsection{Habilidad de descubrimiento: experimentación}

Dyer, Gregersen y Christensen (2012) afirman que los buenos experimentadores saben que el cuestionamiento, la observación y el desarrollo de redes de contactos aportan datos sobre el pasado (qué había) y el presente (qué hay), e infieren que la experimentación es el medio más viable para generar información sobre lo que puede funcionar en el futuro. Así mismo, sostienen que la mayoría de los innovadores utilizan al menos una de las siguientes tres formas de experimentación: la primera consiste en probar nuevas experiencias a través de la exploración, la segunda es desmontar cosas, ya sea física o intelectualmente, y la tercera consiste en materializar una idea a través de pruebas piloto o prototipos. Dentro de los consejos para que el gerente innovador desarrolle las habilidades de experimentación, los autores recomiendan a los gerentes innovadores:

- Cruzar las fronteras físicas: visitar un nuevo país, un departamento distinto de su organización o a una empresa de un sector diferente al suyo y preguntarse: "si tuviera que adoptar algo (un producto, un proceso o cualquier otra cosa) de este entorno en mi entorno habitual, ¿qué copiaría?”.

- Cruzar las barreras intelectuales: suscribirse a un nuevo periódico o revista, o buscar información en Internet de un campo completamente diferente al suyo. 
- Desarrollar una nueva habilidad: para adquirir nuevas perspectivas, necesitamos un plan que nos permita desarrollar nuevas habilidades o adquirir nuevos conocimientos: tomar clases de teatro o fotografía, formarnos en mecánica elemental, electrónica o construcción, o elegir en nuestra empresa un departamento distinto y observar qué podemos aprender sobre su funcionamiento.

- Salir en busca de tendencias: tratar activamente de identificar tendencias emergentes leyendo libros, artículos, revistas, enlaces, blogs y otras fuentes dedicadas a la identificación de nuevas tendencias. Leer material escrito por plumas que destaquen identificando tendencias y apuntando lo que está por venir, luego pensar cómo estas tendencias pueden conducir a un interesante experimento relacionado con un nuevo producto o servicio.

Con este marco teórico, centrado en los autores Dyer, Gregersen y Christensen (2012) y enriquecido con otros autores, esta investigación se dirige a alcanzar el objetivo de distinguir la presencia de las habilidades gerenciales de descubrimiento en instituciones de educación superior de Colombia y Venezuela.

\subsection{Cultura de innovación}

La cultura es el común denominador de las organizaciones, por ello es conveniente denotar que la cultura de innovación forma parte del contexto de la cultura organizacional. En este sentido, se estima necesario abordar la cultura de las organizaciones según algunos autores y sobre tales bases teóricas explicar las concepciones pertinentes a la cultura de innovación.

Dentro de este marco, los autores Beaudan y Smith (2000, citados por Lussier y Achua, 2011) definen la cultura organizacional como la suma total de creencias, actitudes, valores supuestos y formas de hacer las cosas que comparten los miembros de una organización y que se enseña a nuevos miembros. Por su parte, Aguirre (2004) acota que la cultura organizacional es un conjunto de elementos interactivos fundamentales, generados y compartidos por la organización como eficaces para alcanzar sus objetivos, que cohesionan e identifican, por lo que deben ser enseñados a los nuevos miembros.

Bateman y Snell (2009) establecen como cultura organizacional un sistema de valores compartidos sobre lo que es importante para la empresa, incluyendo creencias acerca de cómo funciona el mundo sirviendo de referencia para organizar y guiar el 
comportamiento humano. De igual manera, Robbins y Judge (2013) conceptualizan la cultura como la percepción que comparten todos los miembros de la organización, es decir, un sistema de significados compartidos que distingue una organización de otras, crea un sentimiento de identidad y unidad, enfoca a los empleados en el interés colectivo, fortalece al sistema social, actúa como adherente para mantener a la organización unida y, además funciona como mecanismo de control, moldeando y guiando el comportamiento de los empleados. No obstante, cabe destacar que los elementos comunes en las definiciones sobre cultura organizacional son los valores.

Paredes (2005) define la innovación como un proceso que implica habilidades para llevar a cabo cambios organizaciones y gerenciales, buscando garantizar el éxito comercial de los productos, procesos y servicios. Considera que de existir una cultura de la innovación, ésta tiene necesariamente que estar relacionada con la cultura organizacional. Así mismo, señala que esta atmósfera de interés organizacional por la innovación se concreta en valores y prácticas de sus miembros, que se apoya en lo siguiente:

- La simbiosis cultura organizacional-cultura de la innovación genera un conjunto de valores, creencias, percepciones y prácticas; que como sistema de significados organizacionales, compartidos por los miembros de la organización, configuran y moldean comportamientos pre-activos y reactivos frente a la innovación.

- La práctica de la cultura de la innovación, desarrollada y estimulada por escenarios organizacionales, debe considerar dos situaciones: las relacionadas con la generación de ideas para innovar desde el interior de la organización y las influencias del entorno que viabilizan o no las necesidades del mercado.

En este orden de ideas, Ortega (2007) establece que una cultura de la innovación alienta a las personas a trabajar en innovaciones, porque todos están acostumbrados a sugerir mejoras, modificaciones continuas y replanteamientos institucionales. Además, crea un ambiente al interior de las organizaciones en el que todas las personas se sienten parte activa de las innovaciones y se da una reelaboración colectiva de las informaciones individuales, porque las innovaciones crean nuevas necesidades y abren nuevas perspectivas profesionales. La existencia de una cultura de la innovación y la vivencia que de ella hagan 
todos los miembros de la organización son clave en la generación de estados de ánimo propicios y de entusiasmo, apertura, confianza y colaboración.

Por su parte, Morcillo (2008) establece que la cultura de innovación es una forma de pensar y de actuar que genera, desarrolla y establece valores y actitudes en las organizaciones, propensas a suscitar, asumir e impulsar ideas y cambios que supongan transformación en el funcionamiento y eficiencia de la misma, aun cuando ello implique una ruptura con lo convencional o tradicional. No obstante, la eficiencia y eficacia de un modelo de cultura depende del grado de consenso existente entre los valores, convicciones y patrones de comportamiento adoptados por los diferentes miembros y grupos sociales que componen la organización; estos tendrán que vincularse al estilo corporativo, a la dirección y gestión de los recursos humanos y al aprendizaje organizativo. La innovación es cultura pero, a su vez, la cultura es la "madre' de toda innovación (Cano-Arribi, 2006).

Desde el contexto universitario, se debe acotar que Cebrián (2003) define la innovación educativa como toda acción planificada para producir un cambio en las instituciones educativas que propicie una mejora en los pensamientos, en la organización y en la planificación de la política educativa, así como en las prácticas pedagógicas, y que permitan un desarrollo profesional e institucional con el compromiso y la comprensión de toda la comunidad educativa.

Thomas (2010) advierte que las universidades deben generar innovaciones, si quieren adaptarse a los cambios sociales. Según Cebrián (2003) existen tres claves para los procesos de innovación en la Universidad: la primera se refiere a una atención específica al cambio y a la innovación, ya que actualmente se solicita de la universidad no sólo que genere el conocimiento, sino que construya en su interior una nueva cultura innovadora de la cual se apropie todo el que pasa por ella y así transmitir este conocimiento y cultura a la sociedad. Las otras dos claves, señala el autor, guardan relación con las TIC y están asociadas a la producción de conocimiento y los programas de formación permanente.

Por otro lado, se considera pertinente acotar que, en palabras de Thomas (2009), las universidades aparecen como las instituciones que menos han cambiado con el transcurrir de los siglos, y afirma que la cultura de innovación es un conjunto de asunciones, valores y comportamientos que permiten a un grupo de personas, a un departamento, una facultad 
o una universidad llevar a cabo innovaciones sin mayores resistencias. En este sentido, Clark (1998, citado por Thomas, 2009), señala que el análisis de la actividad innovadora y exitosa en organizaciones de educación superior facilita el desarrollo de una cultura de innovación cuando cuenta con los siguientes elementos: un corpus de gestión potente, una periferia desarrollada y promocionada, una base financiera diversificada, una academia motivada y una cultura emprendedora.

Del mismo modo, Thomas (2009) expone que los primeros cuatro elementos se combinan para crear una cultura que involucra cambio y mantiene los valores fundamentales de la institución, mientras el espíritu emprendedor e innovador puede empezar por un departamento, una institución emprendedora facilita el desarrollo de una cultura que engloba la idea a un nivel institucional. Si bien cada uno de ellos es distinto, las interacciones entre ellos son necesarias para la creación de una institución con cultura innovadora.

Sobre la base de los enfoques teóricos que fundamentan el objetivo de esta investigación, se puede evidenciar la importancia de las habilidades gerenciales para una cultura de innovación. Siendo las cosas así, la figura 3 refleja que dentro de este marco ha de considerarse que la habilidad de un líder con un cargo gerencial puede ser definida como la capacidad de pensar y descubrir ideas creativas, que fungen como estrategias para el establecimiento de valores, creencias, percepciones y actitudes, con el objetivo de que sean utilizadas por los miembros de una organización como herramientas innovadoras, que les permiten obtener resultados eficaces frente a situaciones adversas, y que originan y fortalecen un cultura de innovación. 


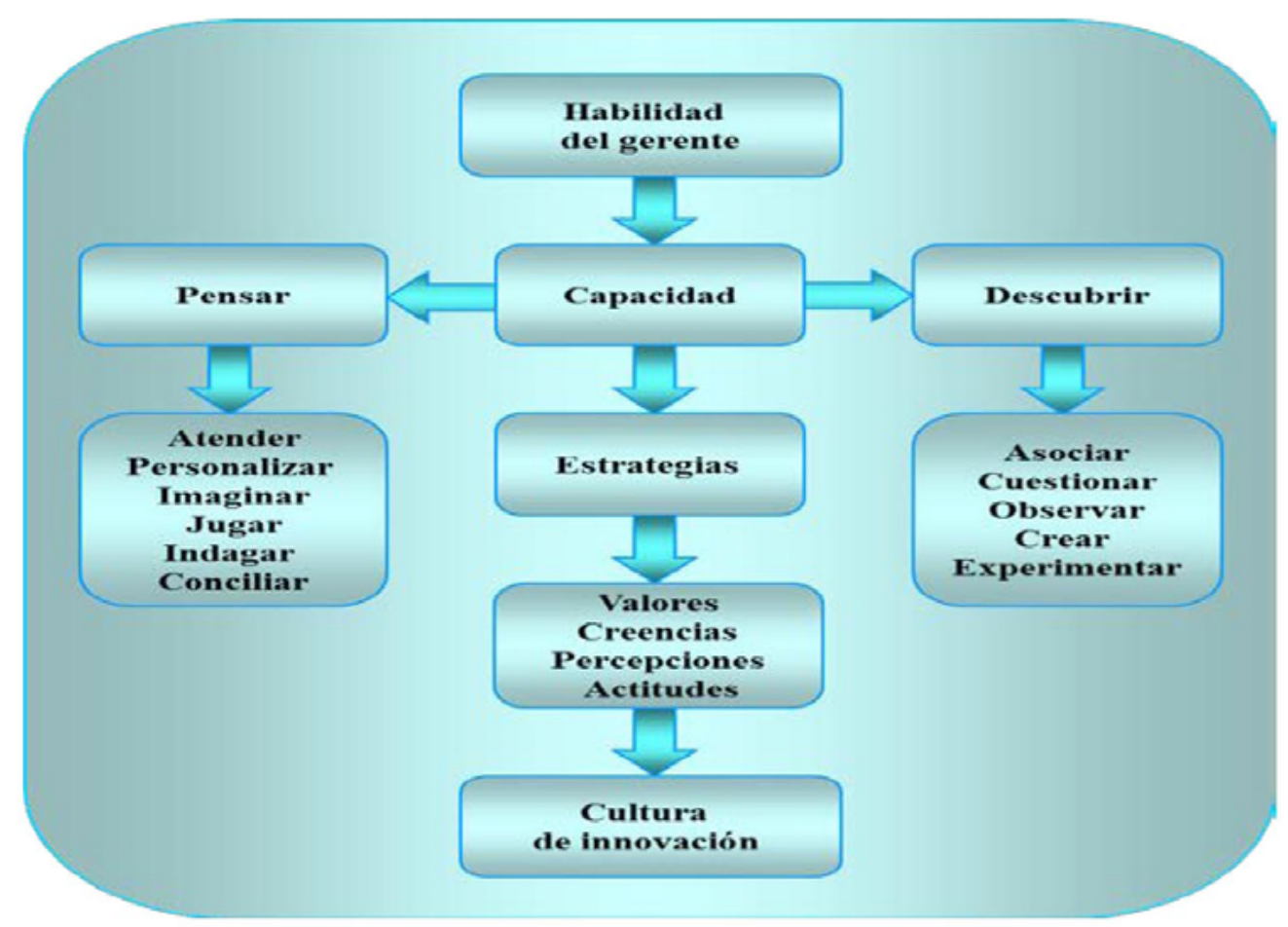

Figura 3. Habilidades gerenciales para una cultura de innovación Fuente: elaborada por los autores.

La capacidad de pensamiento innovador descrita por Horth y Buchner (2009) y la capacidad de descubrimiento creativo explicadas por Dyer, Gregersen y Christensen, (2012), a juicio de esta investigación, son habilidades que deben estar presentes en la gerencia como estrategias de una cultura de innovación.

\section{Metodología}

La presente investigación presenta un enfoque epistemológico bajo el paradigma positivista desde el punto de vista filosófico. Según Corbetta (2007), el estudio de la realidad social se realiza utilizando un marco conceptual, las técnicas de observación y medición para el análisis matemático y los procedimientos de inferencias de las ciencias naturales. Este estudio positivista se orientó a percibir la realidad de manera integral y uniforme, logrando así la cuantificación de los datos para lograr una mayor coherencia, pertinencia y credibilidad (Hernández, Fernández \& Baptista, 2010). 
En opinión de Arias (2012) la investigación descriptiva consiste en la caracterización de un hecho, fenómeno, individuo o grupo, con el fin de establecer su estructura o comportamiento. La investigación fue de tipo descriptivo, pues se identificaron las características del universo de investigación, se señalaron formas de conductas y se establecieron comportamientos concretos (Méndez, 2007).

El diseño de la investigación fue no experimental o expost-facto, lo que, en términos de Kerlinger y Lee (2002), se refiere a cualquier investigación en la que resulta imposible asignar condiciones aleatoriamente a los sujetos; de hecho, no hay condiciones o estímulos a los cuales se expongan los sujetos del estudio. Así mismo, fue transeccional o transversal, como acotan los autores Hernández, Fernández y Baptista (2010), y los datos se recogieron en un solo momento y en un tiempo único, pues el objetivo es describir variables y analizar su incidencia e interrelación en un momento dado.

La investigación fue de campo, dado que la recolección de datos se realizó directamente de la realidad estudiada (Balestrini, 2007), en este caso, la Universidad Antonio Nariño sede Santa Marta, en Colombia y en el Instituto Universitario de Cabimas, en Zulia, Venezuela.

La población estudiada fue de 60 docentes de cada universidad, que sumaron 120. El instrumento de recolección de datos fue un cuestionario de 33 ítems, con categorías de respuesta tipo Likert, validado por expertos, y con una confiablidad de 0,94 bajo el método utilizado del cálculo del coeficiente de Cronbach (alfa).

El procesamiento de los datos implica la forma como se trataron los datos estadísticamente, es decir, el tratamiento estadístico, el cual depende de la naturaleza de la investigación (Chávez, 2007). Esto significa que, tomando en cuenta que la investigación fue de tipo descriptivo, se utilizó como herramienta la estadística descriptiva con las frecuencias absolutas y relativas para procesar el comportamiento de la variable en los distintos sujetos a través del cuestionario. 


\section{Resultados y discusión}

Al identificar las habilidades gerenciales necesarias para ser innovadores en instituciones de educación superior de Colombia y Venezuela, la evidencia presentada en la figura 4 permite apreciar la ausencia de las habilidades gerenciales para una cultura de innovación en las universidades de ambos países. Estos resultados merecen ser referenciados por Horth y Buchner (2009), quienes afirman que la habilidad de prestar atención radica en identificar nuevos patrones de innovación a través de la percepción.

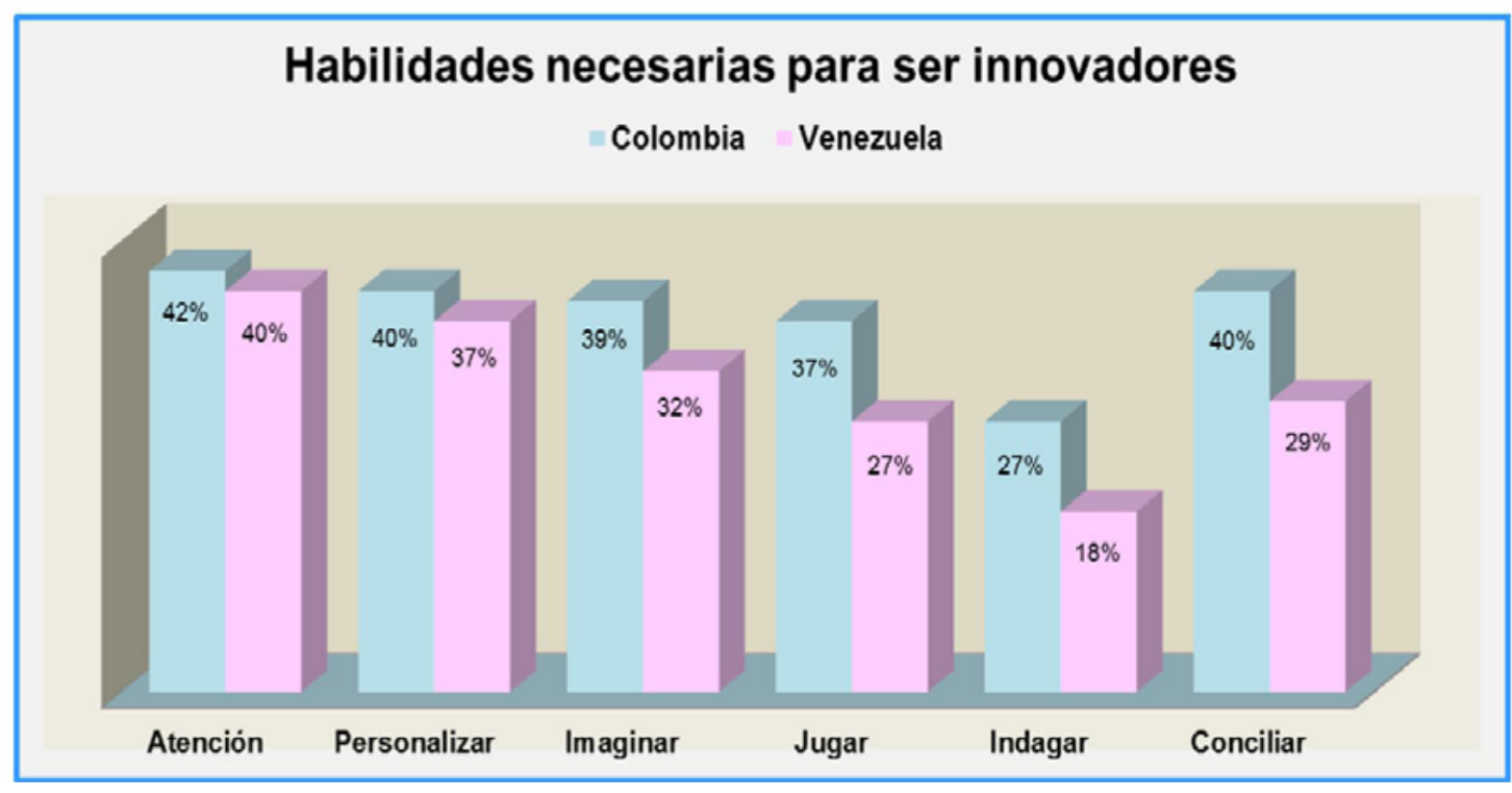

Figura 4. Habilidades gerenciales necesarias para ser innovadores en instituciones de educación superior de Colombia y Venezuela

Fuente: elaborado por los autores.

En este sentido, se infiere que la gerencia de las universidades carece de esa percepción y no posee cultura de innovación para preservar, proteger y fomentar el aprendizaje profundo y amplio para los miembros de las organizaciones, en unas relaciones de atención como lo establecen los autores Hargreaves y Fink (2008). Así mismo, carecen de capacidad para procesar información y abordarla como herramienta para describir situaciones, construir ideas y comunicar de manera eficaz, es decir, no han desarrollado la habilidad de imaginación acotada por Horth y Buchner (2009). 
En lo que se refiere a jugar de manera reflexiva, los resultados reflejan que la gerencia de las universidades investigadas carece de la creación de enfoques mediante la exploración y la experimentación, habilidad necesaria para los innovadores, como lo expresa Buchner (2009). Del mismo modo, impiden la innovación porque no escuchan las ideas, sugerencias y opiniones de sus empleados, consultando decisiones y ofreciendo y solicitando retroalimentación como habilidad para una cultura de innovación (Rodríguez, 2008).

Con respecto a la habilidad de indagar en colaboración con otras personas, los resultados reflejan que para una mayoría significativa de los sujetos encuestados, la gerencia de las universidades no estimula la diversidad, niega que las innovaciones se obtienen a través de un intercambio de ideas y no escucha las opiniones de sus empleados ni consulta sus decisiones. Finalmente, en lo que se refiere a la habilidad de conciliación, los resultados reflejan que para la mayoría de los sujetos encuestados es falso que la gerencia culpe a otros por ser más innovadores, que maneje sus propios errores y que sea ejemplo de creatividad.

Finalmente, se debe acotar que los resultados arrojados por el instrumento para la recolección de la información reflejan la ausencia de las habilidades gerenciales necesarias para ser innovadores en instituciones de educación superior de Colombia y Venezuela.

Desde la otra dimensión, al distinguir la presencia de las habilidades gerenciales de descubrimiento en instituciones de educación superior de Colombia y Venezuela, los resultados reflejados en la figura 5 permitieron poner en evidencia la ausencia de las habilidades de asociación, cuestionamiento, observación, creación de redes y experimentación en la mayoría de los gerentes de las universidades estudiadas. 


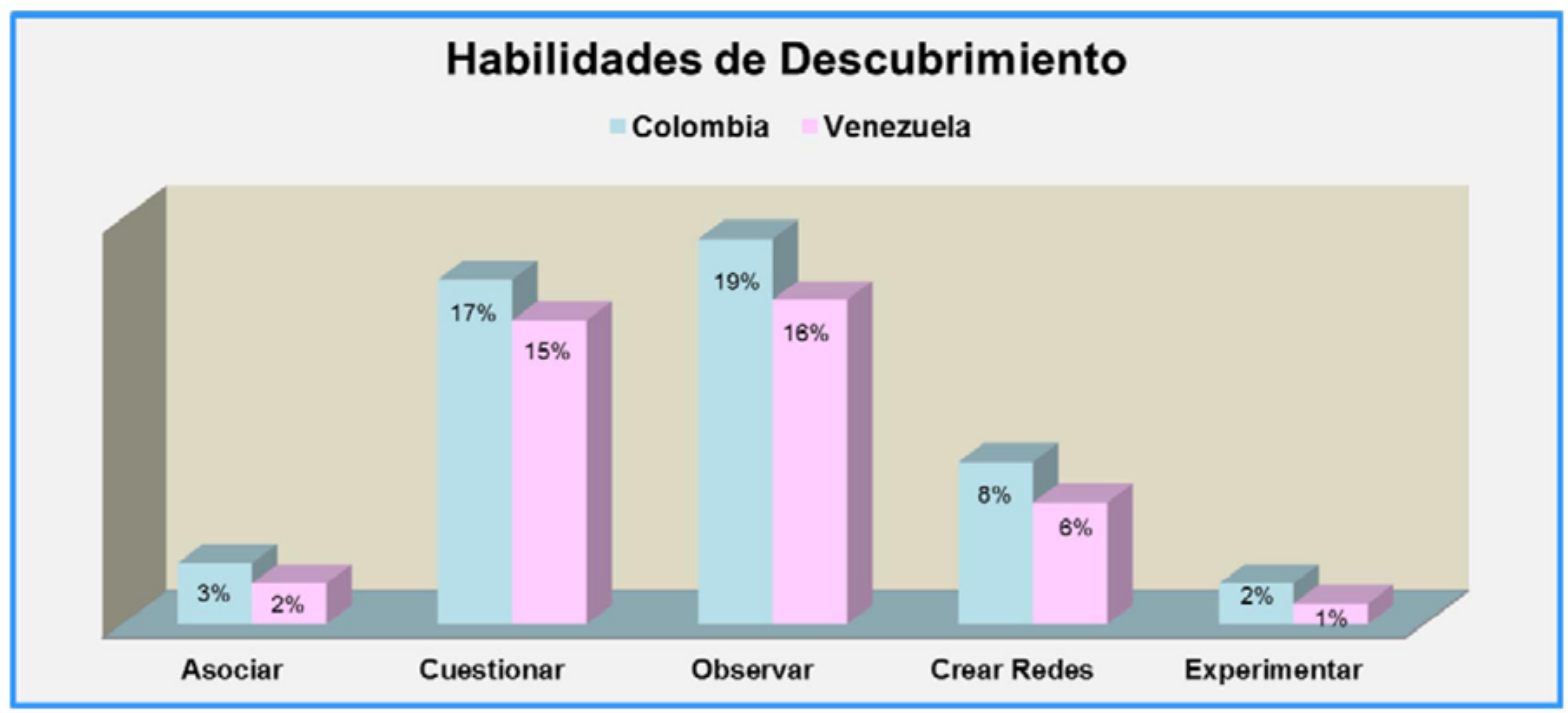

Figura 5. Habilidades gerenciales de descubrimiento en instituciones de educación superior de Colombia y Venezuela

Fuente: elaborada por los autores.

Los resultados demuestran la presencia de la habilidad de asociación en la gerencia de las universidades estudiadas de Colombia y Venezuela solo en $3 \%$ y $2 \%$, respectivamente. Esto significa una marcada ausencia de la capacidad de creatividad para conectar sus experiencias pasadas y de sintetizarlas creando otras nuevas (Dyer, Gregersen \& Christensen, 2012). Así mismo, solo el $17 \%$ de la gerencia en la universidad colombiana y el $15 \%$ de la venezolana admitió la presencia de la capacidad de cuestionar como habilidad de descubrimiento dedicada a la búsqueda de preguntas que invitan a la reflexión, para entender mejor qué es una cosa y lo qué podría ser.

En lo que a la habilidad de observación se refiere, los resultados arrojaron que solo el $19 \%$ de los colombianos encuestados y el $16 \%$ de los venezolanos encuestados admiten la presencia de la habilidad de observación en la gerencia de las universidades, considerando que son intensos observadores del mundo que los rodea, lo que les permite, a su vez, detectar aquello que no funciona (Dyer, Gregersen \& Christensen, 2012).

Del mismo modo, es de observar que los resultados reflejan la creación de redes, como habilidad de descubrimiento en la gerencia de las universidades de Colombia y Venezuela en el $8 \%$ y $6 \%$, respectivamente, es decir, un bajo porcentaje de los encuestados considera 
que la gerencia dedica tiempo y energía a encontrar y probar ideas a través de una red integrada por individuos diversos. Aplica el principio básico de la creación de redes de ideas, construyendo un puente entre diferentes áreas de conocimiento mediante la interacción con las personas.

Finalmente, la habilidad de descubrimiento sobre la experimentación en la gerencia de las universidades estudiadas, a criterio de los encuestados, está presente en porcentajes muy bajos, pues consideran que la gerencia prueba nuevas experiencias a través de la exploración, desmonta cosas, ya sea física o intelectualmente, y se preocupa por materializar una idea a través de pruebas piloto o prototipos, como lo acotan los autores Dyer, Gregersen y Christensen (2012). En este sentido, los resultados arrojados por el instrumento de recolección de datos, permiten inferir la ausencia de las habilidades gerenciales de descubrimiento en instituciones de educación superior de Colombia y Venezuela, acotadas por los autores mencionados.

\section{Conclusiones}

Los resultados arrojados por el cuestionario permiten inferir la ausencia de las habilidades gerenciales necesarias para ser innovadores y de las habilidades gerenciales de descubrimiento. Esto significa una debilidad gerencial, que refleja la incapacidad de pensar y descubrir ideas creativas bloqueando las estrategias para el establecimiento de valores, creencias, percepciones y actitudes como herramientas innovadoras para gestionar una cultura de innovación.

Esta ausencia de las habilidades gerenciales para una cultura de la innovación deja interpretar que en las organizaciones universitarias estudiadas no se fusionan nuevos valores de gestión con los valores académicos tradicionales, de manera que todos los niveles de la institución trabajen para mejorar la cultura académica. No se establecen vínculos entre la academia e industria para generar y promocionar el conocimiento que conlleva el beneficio mutuo ni se buscan formas de compartir los conocimientos y los recursos con los centros educativos vecinos y la comunidad local, lo que significa la ausencia de los elementos de la cultura de innovación. En este sentido, los resultados determinan la ausencia de las habilidades gerenciales para una cultura de innovación universitaria en instituciones de educación superior de Colombia y Venezuela. 


\section{Referencias}

Aguirre, A. (2004). La cultura de las organizaciones. Barcelona: Ariel.

Arias, F. (2012). El proyecto de Investigación: Introducción a la investigación científica (Sexta Edición). Caracas: Episteme C.A.

Balestrini, M. (2007). Cómo se Elabora un Proyecto de Investigación (Quinta Edición). Venezuela: Editorial BL Consultores Asociados.

Barba, E. (2011). Innovación (Segunda Edición). Barcelona: Libros de Cabecera.

Bateman, T., \& Snell, S. (2009). Administración. Liderazgo y colaboración en un mundo competitivo (Octava edición). México: Editorial McGraw-Hill.

Belker, L., \& Topchik, G. (2007). Gerente por primera vez (Quinta Edición). Estados Unidos: Grupo Nelson.

Cano-Arribi, J. (2006). Como crear una cultura de innovación en las organizaciones. España: Editorial McGraw-Hill.

Cebrián, M. (2003). Enseñanza virtual para la innovación universitaria. España: Narcea Ediciones.

Chávez, N. (2007). Introducción a la investigación educativa. Maracaibo: Gráficas González.

Chiavenato, I. (2009). Comportamiento Organizacional: La dinámica del éxito en las organizaciones (Segunda Edición). México: McGraw-Hill.

Chiavenato, I. (2009) Gestión del Talento Humano (Tercera Edición). México: Editorial McGrawHill Interamericana.

Carbetta, P. (2007). Metodología y técnicas de investigación social. España: Editorial McGraw-Hill.

Daft, R. (2015). Teoría y diseño organizacional (11 ${ }^{\mathrm{a}}$ Edición). México: Cengage Learning Editores.

Drucker, P. (2002). De líder a líder. México: Ediciones Granica.

Dyer, J., Gregersen, H., Clayton, M., \& Christensen, C. (2012). El ADN del innovador. Barcelona: Ediciones Deusto S.A.

Gómez, I. (2013). Innovación y cultura organizacional (tesis doctoral). Universidad Nacional de Educación a Distancia, Colombia.

González, J. (2009). Manual de transferencia de tecnología y conocimiento (Segunda Edición). España: Editorial Netbilblo. 
Hargreaves, A., \& Fink, D. (2008). El Liderazgo Sostenible. Siete principios para el liderazgo en centros educativos innovadores. Madrid: Morata.

Hernández, R., Fernández, C., \& Baptista, L. (2010). Metodología de la Investigación (Quinta Edición). México: McGraw-Hill Interamericana.

Hirt, G., \& Ferrel, L. (2010). Introducción a los negocios en un mundo cambiante (Séptima Edición). México: Editorial McGraw-Hill Interamericana.

Horth, D. y Buchner, D. (2009). Liderazgo en materia de innovación. Cómo utilizar la innovación para liderar con eficacia, trabajar en colaboración y obtener resultados. Bruselas: Editorial Center for Creative Leadership.

Ivancevich, J., Konopaske, R., \& Matteson, M (2006). Comportamiento organizacional (Séptima Edición). México: Editorial McGraw-Hill Interamericana.

Kerlinger, F., \& Lee, H. (2002). Investigación del comportamiento (Cuarta edición). México: Editorial McGraw-Hill.

Koontz, H., Weihrich, H., \& Cannice, M. (2012). Administración. Una perspectiva global y empresarial (14 ${ }^{\mathrm{a}}$ Edición). México: Editorial McGraw-Hill.

Lara, A. (2012). Desarrollo de habilidades de pensamiento y creatividad como potenciadores de aprendizaje. Revista Unimar Colombia, (59), 85-96.

López, R. (2016). Diccionario de Creatividad. Conceptos y Expresiones para comprensión de la creatividad (Quinta Edición). Santiago de Chile: Editorial Universitaria.

Lussier, R., \& Achua, C. (2011). Liderazgo. Teoría, aplicación y desarrollo de habilidades (Cuarta Edición). México: Cengage Learning Editores S.A.

Madrigal, B. (2009). Habilidades Directivas (Segunda Edición). México: Editorial McGraw-Hill.

Méndez, C. (2007). Metodología, diseño y desarrollo del proceso de investigación con énfasis en ciencias empresariales (Cuarta edición). Colombia: Editorial Limusa

Mintzberg, H. (1998). El proceso estratégico. Madrid: Prentice Hall.

Morcillo, P. (2008). Innovación, a por todas con la cultura. Madrid. Innovación sin fronteras, 20(1), 66-76.

Morrisey, G. (1996). Pensamiento Estratégico. México: Prentice Hall.

Murcia, H. (2010). Creatividad e innovación para el desarrollo empresarial. Colombia: Ediciones de la U.

O’Guinn, T., \& Semenick, R. (2004). Publicidad y Comunicación integral de marca. México: International Thompson Editores. 
Ohmae, K. (2007). La Mente del Estratega. México: McGraw-Hill.

Ortega, P. (2007). Modelo de innovación educativa. Un marco para la formación y el desarrollo de una cultura de la innovación. México: AIESAD RIED.

Paredes, L. (2005). Los factores incidentes en el comportamiento innovativo de las empresas mediana. Espacios, 17(2). Recuperado de http://www.revistaespacios.com/ a96v17n02/30961702.html

Pérez, M. (2013). La creatividad en la docencia universitaria. Cuaderno de Pedagogía Universitaria. Año 10, (19), 2-6.

Robbins, S. (2004). Comportamiento Organizacional (Novena Edición). México: Prentice Hall.

Robbins, S., \& Judge, T. (2013). Comportamiento Organizacional. (15ª Edición). México: Editorial Pearson.

Robert, M. (2006). El Nuevo Pensamiento Estratégico. México: McGraw-Hill.

Rodríguez, M. (2008). Formación Gerencial en Valores. Bogotá: Editorial Universidad Nacional de Colombia.

Senge, P. (1994). La quinta disciplina: cómo impulsar el aprendizaje en la organización inteligente. Barcelona: Granica.

Tomas, M. (2009). La Cultura Innovadora de las Universidades. Barcelona: Editorial Octaedro.

Tomas, M. (2010). La cultura innovadora de las universidades (Segunda edición). Barcelona: Ediorial Octaedro.

Waisburd, G. (1 de diciembre de 2009) Pensamiento creativo e innovación. Revista Digital Universitaria, 10(12). Recuperado de: http://www.revista.unam.mx/vol.10/num12/ art87/int87.htm

Whetten, D., \& Cameron, K. (2005). Desarrollo de habilidades directivas (Sexta Edición). México: Editorial Pearson Educación.

Whetten, D., \& Cameron, K. (2011). Desarrollo de habilidades directivas (Octava Edición). México: Editorial Pearson Educación. 